\title{
Estrategias para la gestión del talento humano en las asociaciones de yuqueros adscritas a Colfeyuca en Sucre, Colombia
}

\section{Strategies for the management of human talent in the associations of yuqueros attached to Colfeyuca in Sucre, Colombia}

\author{
Diego Cardona-Arbeláez ${ }^{1}$ \\ Julieth Cecilia Alzate-Alvarán² \\ Harold Lora-Guzmán ${ }^{3}$ \\ Recibido: diciembre 09 de 2017 \\ Aceptado: abril 21 de 2018
}

\section{Resumen}

Este artículo tiene como objetivo identificar las estrategias para la gestión del talento humano que actualmente desarrollan las asociaciones yuqueras del departamento de Sucre, Colombia. El análisis se realiza a la luz de la teoría de la responsabilidad social empresarial, tomando como base los indicadores del instituto Ethos, de Brasil. La investigación tiene un enfoque cualitativo, en la cual se desarrollaron entrevistas a profundidad con los líderes de diez asociaciones de cultivadores de yuca; así mismo, la observación fue parte importante del estudio, lo cual complementó el análisis de la información. Los resultados indican que las asociaciones, a pesar de estar constituidas legalmente, no adelantan acciones en pro de la gestión del talento humano, y esto se da básicamente por desconocimiento sobre el tema. Sin embargo, existe intención de cambio, porque las asociaciones reconocen la importancia que tiene el talento humano dentro de las empresas. Esto las ha llevado a unirse y gestionar capacitaciones y recursos de forma conjunta, para favorecer el crecimiento gremial.

Palabras Clave: talento humano, responsabilidad social empresarial, gestión, capacitación.

\begin{abstract}
The objective of this article is to identify strategies for the management of human talent that are currently being developed by the yuqueras associations of the department of Sucre, Colombia. The analysis is carried out in light of the theory of corporate social responsibility, based on the indicators of the Ethos Institute in Brazil. The research has a qualitative approach, in which in-depth interviews were conducted with the leaders of ten associations of cassava growers; Likewise, the observation was an important part of the study, which complemented the analysis of the information. The results indicate that the associations, in spite of being constituted legally, do not advance actions in favor of the management of human talent, and this is basically due to ignorance on the subject. However, there is an intention to change, because associations recognize the importance of human talent within companies. This has led them to join and manage training and resources jointly, to promote union growth.
\end{abstract}

Keywords: human talent, corporate social responsibility, management, training.

1 Administrador de Empresas, Magíster en Desarrollo Empresarial, Estudiante del Doctorado en Administración - Universidad del Norte-, Universidad Libre, Cartagena, Colombia. E-mail: diego.cardona@unilibrectg.edu.co

2 Administradora de Negocios Intternacionales, Magíster en Administración, Becaria programa de formación de alto nivel del departamento de Sucre -Alcaldía de Sincelejo-, Sincelejo, Colombia. E-mail: jcalzate_alvaran@hotmail.com

3 Ingeniero de Productividad y Calidad, Doctor en Cultura y Educación, Universidad de Cartagena, Cartagena, Colombia. E-mail: hlorag@ unicartagena.edu.co 


\section{Introducción}

En este siglo, la Gestión del Talento Humano, GTH, ha llegado a ser reconocida como una parte inherente a la administración de las organizaciones, donde lo que se persigue es mejorar y mantener relaciones integrales en todas las áreas de la empresa (Alean-Pico, del Río, Simancas-Trujillo \& Rodríguez-Arias, 2017). Esto permite promover el alcance de los objetivos de la organización, garantizando la eficacia y el máximo desarrollo de los recursos humanos a través del trabajo en equipo de las distintas áreas (Vergara-Arrieta, 2015). Para ello, se debe tener en cuenta la importancia de crear un equilibrio entre los objetivos individuales de los empleados y los de la organización.

En el ámbito teórico, se han identificado varias posturas sobre la GTH: Iglesia-Navas, Rosero-Flórez y Castañeda-Villacob (2018), señalan que hablar de administración de las personas es hablar de gente, de inteligencia, de vitalidad, de mente, de acción y de proacción; mientras que Stoner, Freemany Gilbert (1996), la delimitan a una estrategia para lograr la eficiencia. Asimismo, Rojas-Moreno y Alonzo (2015), Citanto a Elton Mayo, destacan la teoría de las relaciones humanas vista desde una óptica humana, por lo que modifica el modelo mecánico del comportamiento organizacional para sustituirlo por otro que tuviese más en cuenta los sentimientos, actitudes, complejidad motivacional y otros aspectos del sujeto.

Otra visión es la de Vásquez-Bustos (2013), señalando a la GTH como una herramienta estratégica de dirección, cuyo objetivo es obtener la máxima creatividad. Esto se alcanza a través de un conjunto de acciones dirigidas a disponer en todo momento del nivel de conocimiento, capacidades y habilidades en la obtención de los resultados necesarios para ser competitivo en el entorno actual y futuro (Romero-González, 2014; Parra-Penagos \& Rodríguez-Fonseca, 2016).

La Responsabilidad Social Empresarial, RSE, busca generar bienestar para todas las partes interesa- das, stakeholders en inglés (García-Rubiano \& Forero-Aponte, 2015). Por ello, si la gerencia adopta esta filosofía, las empresas podrán beneficiarse de un talento humano más comprometido y convencido de los mismos lineamientos que declara la RSE (del Río-Cortina, Cardona-Arbeláez \& Guacarí-Villalba, 2017). En este sentido, será posible hablar de desarrollo humano y social, en la medida en que se atienden los derechos de las personas (Vergara-Arrieta \& Carbal-Herrera, 2014).

El talento humano en la organización, visto desde la RSE, es uno de los stakeholders más importantes, ya que con ellos es posible alcanzar los objetivos que se proponga la empresa; además, es el que conforma el primer círculo de confianza de cualquier organización (Balza-Franco \& Cardona-Arbeláez, 2015). Teniendo en cuenta la evolución constante del comportamiento humano, y que las organizaciones día tras día buscan ser más productivas y competitivas, es importante analizar los aspectos que permitan convertir en una estrategia la GTH, ya que se trata de un factor primordial y diferenciador frente a la competencia (Morales-Rubiano, Ortiz-Riaga, Duqe-Orozco \& Plata-Pacheco, 2017; Alzate, 2017).

Este artículo tiene como objetivo identificar las estrategias para la GTH que actualmente desarroIlan las asociaciones yuqueras del departamento de Sucre, Colombia. El análisis se realiza a la luz de la teoría de la RSE, tomando como base los indicadores del instituto Ethos. Se inicia con una construcción cronológica de las diferentes teorías sobre el desarrollo de la GTH, que sirve como base para analizar las acciones y/o prácticas de las asociaciones en el campo económico de estudio. Los resultados permiten brindar algunas recomendaciones para convertir a la GTH en una estrategia que genere beneficios para las asociaciones.

\section{Metodología}

La investigación se adelantó bajo el marco de un enfoque cualitativo, como lo indican Bonilla-Castro, Hurtado-Prieto y Jaramillo-Herrera (2009). Los 
métodos, técnicas e instrumentos de recolección de información, fueron de fuentes primarias, correspondientes a entrevistas semiestructuradas realizadas a los representantes legales de las asociaciones de yuqueros de Sucre. Las entrevistas se realizaron con un estilo informal, cuestionarios administrados, que permitieron ver situaciones nuevas para generar preguntas a hechos inusuales que se dan dentro de las instituciones (Silva-Cañaveral, 2016). Igualmente, se utilizaron fuentes secundarias como: estudios de RSE, entrevistas, documentos, libros, artículos de internet y registros, entre otros.

La población correspondió a las asociaciones de yuqueros adscritas a la Federación Colombiana de Yuca, Colfeyuca, del departamento de Sucre. En ella se trabajó con una muestra de 10 asociaciones, las cuales fueron seleccionadas por su disponibilidad para participar en la investigación.

La recolección de información se llevó a cabo en 4 fases. La primera consistió en el desarrollo de una reflexiona cerca de la temática, teniendo en cuenta el medio socioeconómico del departamento de Sucre, para lo cual se visitaron las asociaciones. En la segunda fase se desarrolló el trabajo de campo en el que se propició un diálogo abierto entre las partes, lo cual permitió contextualizar el perfil de las asociaciones objeto de estudio. En la tercera fase se codificó el cuestionario administrado y se procedió a analizar la información obtenida. En la cuarta fase se dieron a conocer los resultados a las asociaciones participantes, para recibir la retroalimentación del proceso.

Las entrevistas a profundidad se realizaron con los representantes legales de cada asociación, y la información recolectada fue analizada con el software Atlas Ti (Medina-Monterrosa \& Carvajalino-Slaghekke, 2014). En el análisis de los procesos de gestión del talento humano al interior de las asociaciones, se tuvieron en cuenta las consideraciones de Mondy (2010), las cuales se enlistan en la tabla 1.

\begin{tabular}{ll}
\hline Parámetros & Descriptor \\
\hline Consideraciones éticas, sociales y legales & $\begin{array}{l}\text { Ética empresarialy responsabilidad social corporativa } \\
\text { Diversidad de la fuerza laboral, oportunidad equita- } \\
\text { tiva de empleo y acción afirmativa } \\
\text { Análisis de puestos, planeación estratégica y planea- } \\
\text { ción de los recursos humanos }\end{array}$ \\
Rotación de personal & Reclutamiento \\
Selección & $\begin{array}{l}\text { Capacitación y desarrollo } \\
\text { Planeación y desarrollo de la carrera }\end{array}$ \\
Remuneración & $\begin{array}{l}\text { Administración y evaluación del desempeño } \\
\text { Remuneración financiera directa }\end{array}$ \\
Seguridad y salud & $\begin{array}{l}\text { Prestaciones, recompensas no financieras y otros as- } \\
\text { pectos de la remuneración } \\
\text { Un ambiente laboral seguro y saludable }\end{array}$
\end{tabular}




\begin{tabular}{ll}
\hline Parámetros & Descriptor \\
\hline Relaciones con los empleados y relaciones laborales & Sindicatos laborales y negociaciones colectivas \\
& $\begin{array}{l}\text { Relaciones internas con los empleados } \\
\text { La operación en un ambiente global }\end{array}$ \\
& $\begin{array}{l}\text { Administración global de recursos humanos } \\
\text { La aplicación del instrumento Ethos y el proceso de } \\
\text { validación en el software }\end{array}$ \\
\hline
\end{tabular}

Tabla 1. Elementos que caracterizan la administración de los recursos humanos.

La información se obtuvo a través de cuestionarios administrados: uno basado en la propuesta del Instituto Ethos, de Brasil; mientras que el otro cuestionario se basó en una encuesta de la Asociación Nacional de Industriales, ANDI (Coba-Molina, Díaz-Córdova, Zurita-Meza \& Broaño-López, 2017). Estos cuestionarios se adaptaron, siendo validados con el software estadístico SPSS.

El análisis de fiabilidad del instrumento, con 22 elementos, arrojó un Alpha de Cronbach de 0,808. Teniendo en cuenta que el criterio de evaluación es que este valor sea superior a 0,7 , se concluye que el instrumento de medición es adecuado en cuanto a su consistencia interna, siendo útil para su aplicación.

\section{Resultados y discusión}

El análisis del cuestionario permitió identificar seis etapas o estadíos, en los que se pueden encontrar las asociaciones. La primera etapa señala las acciones básicas de la compañía, en la cual está todavía en nivel reactivo a las exigencias legales. La segunda etapa representa una fase intermediaria de acciones, en la cual la compañía mantiene una postura defensiva sobre los temas, pero ya empieza a gestionar cambios y avances respecto a la conformidad de sus prácticas. La tercera etapa es una fase avanzada de acciones, en la cual ya se reconocen los beneficios de ir más allá de la conformidad legal, preparándose para nuevas presiones reglamentadoras en el mercado, de la sociedad, etc. En esta etapa, la responsabilidad social y el desarrollo sostenible, son considerados estratégicos para el negocio. La cuarta etapa es una fase proactiva, en la cual la compañía alcanza estándares considerados de excelencia en sus prácticas, involucrando a proveedores, consumidores, clientes, a la comunidad y también influenciando políticas públicas de interés de la sociedad. Finalmente, las etapas 5 y 6 corresponden al caso en que la empresa no se ubique en ninguna de las anteriores.

La información recolectada permitió establecer que las asociaciones yuqueras estudiadas, en cuanto al público interno, enmarcado en: los empleados, sus familias y las condiciones laborales, entre otras, presenta la siguiente tendencia.

Diálogo y participación: en cuanto a la gestión participativa, un $50 \%$ de las asociaciones considera que la información sobre la compañía está a disposición de los empleados, como se indica en la figura 1. Sin embargo, resulta bastante llamativo que un $40 \%$ considera que nunca han tratado ese asunto antes. Esta disparidad lleva a pensar que aquellas asociaciones que no se encuentran en la etapa 1, es por la sencilla razón de no tener clara la concepción de que los empleados participan de las decisiones que se toman al interior de la asociación. Lo anterior se infiere de las entrevistas informales que se dieron con las diferentes personas, todas acertaban en sentirse parte de la empresa y estar enterados de lo que sucedía. 


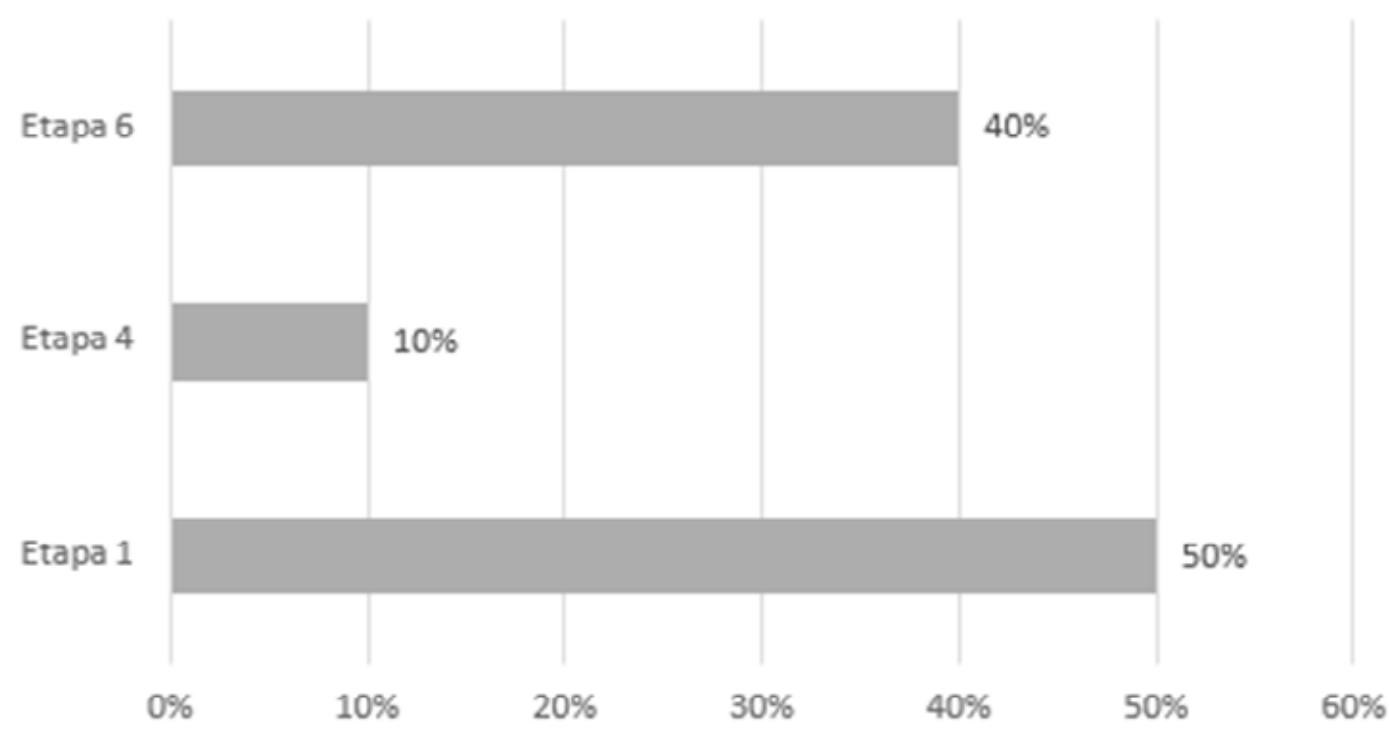

Figura 1. Diálogo y participación.

Respeto al individuo: este aspecto corresponde al compromiso con el futuro de los niños, Compromiso con el desarrollo infantil, Valoración de la diversidad, Compromiso con la no discriminación y promoción de la equidad racial, Compromiso con la equidad de género. Atendiendo a la figura 2, este indicador que considera los elementos en los que se involucra a los empleados en la gestión de la empresa, tuvo un pobre desempeño. Es decir, que, a pesar de su importancia, este tema aún no ha sido considerado por la mayoría de las asociaciones. Igualmente, en lo referente al tema de los niños, las asociaciones se encuentran en la etapa 1 ; es decir, se da el respeto por la legislación nacional en cuanto al tema del compromiso con el futuro de los niños. Sin embargo, las asociaciones no tienen establecidos compromisos en pro del desarrollo infantil, ni han tratado con anterioridad los temas de los otros tres indicadores, que pertenecen a este subtema. En síntesis, es bastante insuficiente y prácticamente nulo el avance en lo relacionado con los indicadores de respeto al individuo. 


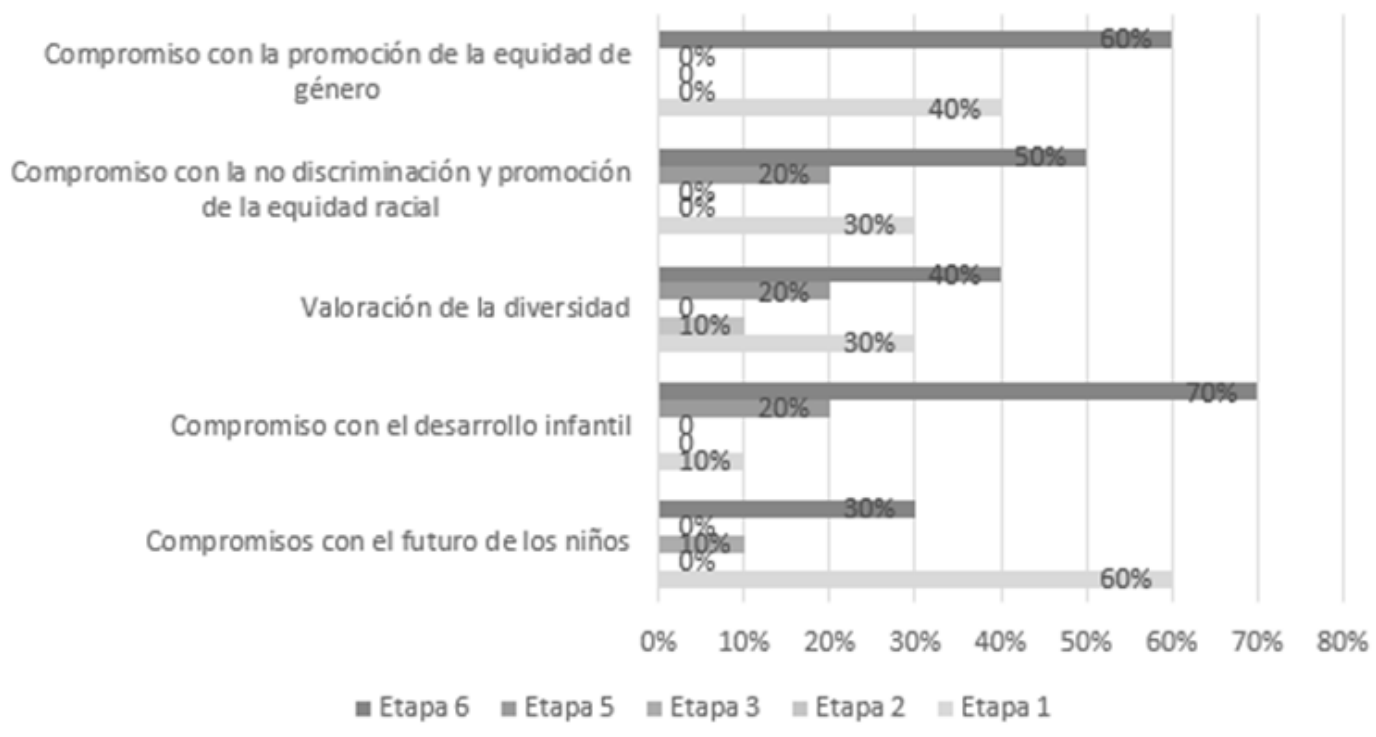

Figura 2. Respeto al individuo.

Trabajo decente: incluye los indicadores de política de remuneración, prestaciones y carrera, Cuidados con salud, seguridad y condiciones de trabajo y preparación para la jubilación. En la figura 3 se ilustran los resultados de este aspecto, encontrán- dose en una situación crítica. Esto debido a que las asociaciones no consideran la aplicación de estos temas o nunca han tratado el asunto con anterioridad.

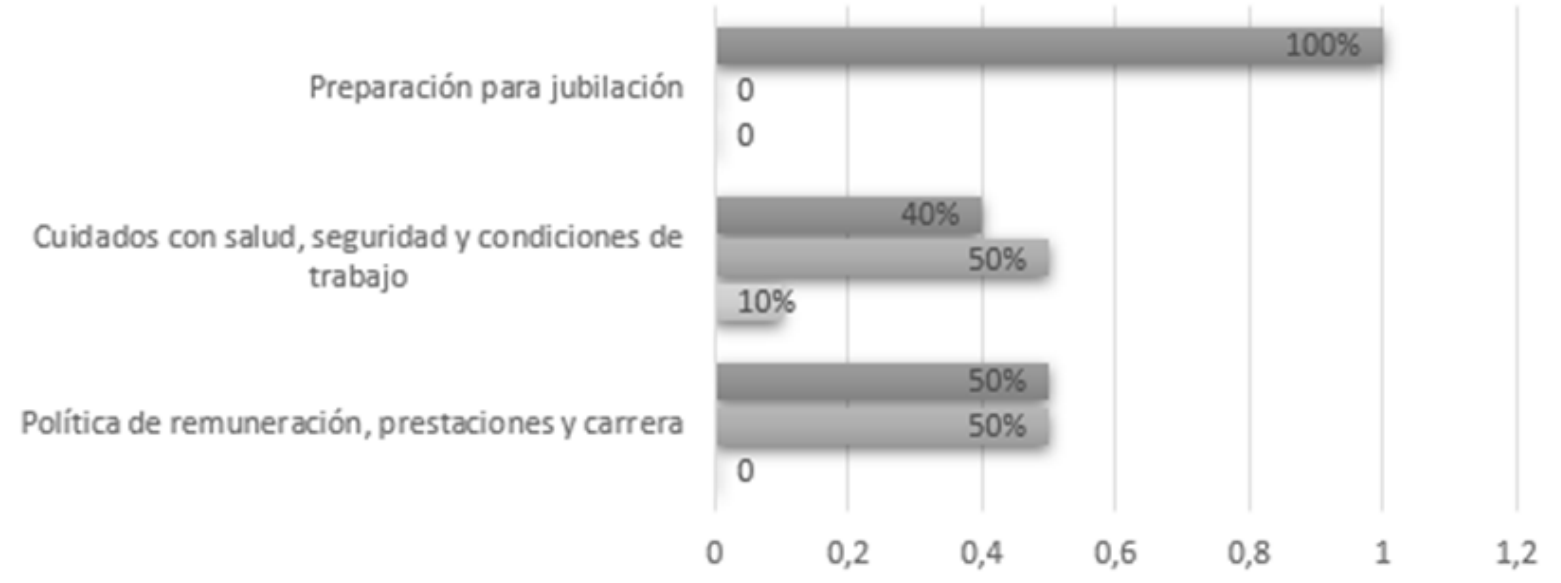

Etapa 6 Etapa 5 Etapa 1

Figura 3. Trabajo decente. 
En cuanto a las medidas de protección de los empleados, se logró establecer que este es un tema que no se está implementando al interior de las organizaciones de yuqueros objeto de estudio. Sin embargo, se evidenció el interés de las asociaciones por implementar estos procesos, en gran medida por las exigencias legales que impone la legislación colombiana; especialmente aquellas que implican que todo empleado debe laborar bajo las medidas de protección acordes a las necesidades de su actividad. Ejemplo de ello fue lo comentado por el señor Mario Enamorado, asociado de la Cooperativa Agroindustrial de Los Palmitos: "Bueno, acá lo que se contrata es por labor... ellos saben que deben venir vestidos para que el sol no los maltrate".

En cuanto a la inclusión del género femenino, los resultados revelaron que las asociaciones consideran que los trabajos que se realizan en la producción y comercialización de yuca, corresponden a acciones laborales muy pesadas para las mujeres. Esto explica el por qué las mujeres, en el sector yuquero, solo se dedican a labores básicas, como lo afirmó Luis Eduardo Herazo, asociado de Asoagroconcepción: "Las mujeres en sí se dedican a repartir refrigerios o a colaborar haciendo comidas o picando yuca, si es el caso de que la asociación se dedique a la venta de chips de yuca".

En lo relacionado con la jubilación, se encontró una de las principales debilidades del sector yuquero de Sucre, debido a que ninguna de las asociaciones realiza los aportes de ley. Estos aportes son fundamentales para que los trabajadores puedan acceder a la pensión, lo que garantiza una vejez digna. Luego de haber laborado durante toda su vida, lo menos que espera un trabajador es llegar a la edad adulta y contar con los recursos necesarios para suplir sus necesidades básicas.

Tampoco se cuenta con afiliaciones a: salud, pensión, riesgos laborales ni caja de compensación. Esto indica el alto grado de informalidad en el que viven los trabajadores, lo cual indica que el sector yuquero debería ajustarse, por lo menos, a la le- gislación vigente en el país. Esta situación de informalidad es común al sector agrícola tanto en Colombia como en Latinoamérica, lo cual limita el nivel de vida de las personas que laboran en este sector (Hernández-Laos, 2013).

Es fundamental comprender que la focalización en el factor humano, entendido como: las capacidades, las habilidades y el mejoramiento de prácticas y relaciones con el entorno, es la base para garantizar las condiciones del bienestar humano, tanto a nivel interno como para el contexto social de una empresa. Las asociaciones no pueden desconocer el compromiso que adquieren con cada empleado, al momento de concertar algún tipo de labor. Esto se considera un contrato laboral, teniendo en cuenta que existe una relación de subordinación y el desarrollo de unas actividades específicas, durante cierto tiempo, y en condiciones determinadas (Gómez-Vélez, 2014).

En vista de lo anterior, algunas de las siguientes consideraciones podrían ser tenidas en cuenta por el Área de Gestión del Talento Humano de las organizaciones, para mejorar en gran medida su accionar respecto a su capital humano:

i) Brindar oportunidades de crecimiento: brindar capacitación a los empleados para que estos despierten su interés por el crecimiento personal y profesional, así como el de sus familias.

ii) Generar canales de Comunicación para recibir sugerencias y opiniones: establecer un buzón de sugerencias o prácticas mensuales de reunión para conocer las sugerencias y opiniones de los empleados.

iii) Priorizar la contratación de personal de la comunidad: se debe priorizar la contratación de personal de la zona para la ejecución de actividades propias del negocio; esto promoviendo la oferta de mano de obra calificada. 
iv) Contribuir a la calidad de vida de las familias de los empleados: conocer a través de un profesional especializado, el ambiente psicosocial de los empleados y sus familias. De esta forma diseñar estrategias tendientes a contribuir en la medida de las posibilidades a mejorar las condiciones de vida.

v) Conocer y apropiarse de las buenas prácticas medioambientales: establecer un cronograma de capacitaciones en torno al tema medioambiental para los empleados, para que sean puestas en práctica al momento de realizar las labores propias de la actividad económica de la asociación.

En síntesis, esta investigación constituye un aporte valioso para el departamento de Sucre, teniendo en cuenta que es la primera que se realiza sobre la Responsabilidad Social Empresarial en torno a las asociaciones de yuca adscritas a Colfeyuca. En este sentido, se espera que los hallazgos sobre el estado actual de la GTH en las asociaciones, sirva como base para proponer políticas que permitan gestionar el talento humano con una visión de responsabilidad social en las organizaciones.

\section{Conclusiones}

Los resultados indican que las asociaciones objeto de estudio, a pesar de estar constituidas legalmente, en la actualidad desconocen las obligaciones legales para con sus empleados. Esto resulta preocupante ya que, desde la visión empresarial y de crecimiento económico que las asociaciones quieren tener, deberían cumplir con los aspectos mínimos de ley.

En general, las asociaciones poseen un alto potencial para generar el cambio en la Gestión del Talento Humano, GTH, ya que tienen intención de mejoramiento. El interés de cambio las ha llevado a unirse, gestionando capacitaciones y recursos en pro del crecimiento gremial. En este sentido, se considera pertinente que desde Colfeyuca se maneje un área de GTH, que sea el eje central para todas las asociaciones. Esto permitirá que aspectos contemplados en la legislación colombiana, como: pago de aportes parafiscales, dotación y jornadas laborales, entre otros, puedan ponerse en práctica para beneficio de las organizaciones y sus asociados.

Finalmente, las consideraciones éticas, sociales y legales que orientan la GTH, deberían llevar a las asociaciones a ejecutar acciones que contemplen aspectos como: dotación de personal, desarrollo del recurso humano, remuneración adecuada, seguridad y salud, relaciones con los empleados y relaciones laborales, así como la operación en un ambiente global.

\section{Referencias}

Alean-Pico, A., Del Rio, J., Simancas-Trujillo, R., \& Rodríguez-Arias, C. (2017). ¿El emprendimiento como estrategia para el desarrollo humano y social?. Saber, Ciencia Y Libertad, 12 (1). Recuperado de: http://www.sabercienciaylibertad.org/ojs/index.php/scyl/article/view/218

Alzate, J. (2017). La responsabilidad social empresarial en el marco del desarrollo sostenible: una mirada desde las asociaciones de yuca del departamento de Sucre, adscritas a Colfeyuca. Cartagena, Colombia.

Balza-Franco, V. B., \& Cardona-Arbeláez, D. (2015). La Responsabilidad Social Empresarial Y La Lucha Contra La Pobreza. Saber, ciencia y libertad, 10 (1), 115-124. doi: http://dx.doi.org/10.22525/sabcliber.2015v10n1.1151

Bonilla-Castro, E., Hurtado-Prieto, J., \& Jaramillo-Herrera, C. (2009). La investigación. proximaciones a la costrucción del conocimiento científico. Bogotá, Colombia: Alfaomega.

Coba-Molina, E., Díaz-Córdova, J., Zurita-Meza, E., \& Proaño-López, P. (2017). La responsabilidad social empresarial en las empresas del Ecuador. Un test de relación con la imagen corporativa y desempeño financiero. Ingeniería Industrial: Actualidad y Nuevas Tendencias, 5 (18), 23-44. Recuperado 
de: http://servicio.bc.uc.edu.ve/ingenieria/revista/ Inge-Industrial/volv-n18/art02.pdf

Del Río-Cortina, J., Cardona -Arbeláez, D., \& Guacarí-Villalba, A. (2017). Responsabilidad social empresarial y construcción de la marca: una nueva mirada a las estrategias de gestión. Revista de Investigación, Desarrollo e Innovación, 8 (1), 49-60. doi: https://doi.org/10.19053/20278306. v8.n1.2017.7370

García-Rubiano, M., \& Forero-Aponte, C. (2015). Contrato psicológico y cambio organizacional en una entidad perteneciente al sector terciario de la ciudad de Bogotá, Colombia. Revista de Investigación, Desarrollo e Innovación, 6 (1), 15-28. doi: http://doi.org/10.19053/20278306.4047

Gómez-Vélez, M. A. (2014). Sobre la flexibilidad laboral en Colombia y la precarización del empleo. Diversitas: Perspectivas en Psicología, 10 (1), 103116. Recuperado de: http://www.redalyc.org/articulo.oa?id=67935714007

Hernández-Laos, E. (2013). Legislación laboral, sector informal y productividad multifactorial en México. Economía UNAM, 10 (28), 05-52. Recuperado de: http://www.scielo.org.mx/scielo.php?script=sci_arttext\&pid=S1665-952X2013000100002\&ln$\mathrm{g}=\mathrm{es} \&$ tlng=es.

Iglesia-Navas, M. A., Rosero-Flórez, K., \& Castañeda-Villacob, J. H. (2018). La gestión del talento humano y su relación con la innovación en las pymes de la industria de alimentos en Barranquilla, Colombia. Revista Espacios, 39 (06), 3. Recuperado de: http://www.revistaespacios.com/ a18v39n06/18390603.html

Medina-Monterrosa, P., \& Carvajalino-Slaghekke, A. (2014). Ni al tigre ni al cuero: la toma de decisiones y el uso los sistemas de soporte y de ayuda a la decisión SSD-SAD. Saber, Ciencia y Libertad, 9 (1), 115-128. Recuperado de: http://www.saber- cienciaylibertad.com/ojs/index.php/scyl/article/ view/26

Mondy, R. W. (2010). Administración de recursos humanos. México: Pearson Educación.

Morales-Rubiano, M., Ortiz-Riaga, C., Duque-Orozco, Y., \& Plata-Pacheco, P. (2017). Fuentes de conocimiento e imágenes de la innovación en micro y pequeñas empresas de turismo: agencias de viajes y hoteles en Bogotá y Pereira. Revista de Investigación, Desarrollo e Innovación, 7 (2), 217-230. doi: https://doi.org/10.19053/20278306.v7.n2.2017.6081

Parra-Penagos, C., \& Rodríguez-Fonseca, F. (2016). La capacitación y su efecto en la calidad dentro de las organizaciones. Revista de Investigación, Desarrollo e Innovación, 6 (2), 131-146. doi: http://dx. doi.org/10.19053/20278306.4602

Rojas-Moreno, I., \& Alonzo, B. (2015). Administración de recursos humanos (teorías, escuelas y análisis). Recuperado de: http://www.academia. edu/13621942/ADMINISTRACI\%C3\%93N_DE_RECURSOS_HUMANOS_TEOR\%C3\%8DAS_ESCUELAS_Y_AN\%C3\%81LISIS_

Romero-González, Z. (2014). El sistema de aseguramiento de la calidad en la educación superior. Saber, Ciencia y Libertad, 9 (2), 11-14. doi: http://dx. doi.org/10.22525/sabcliber.2014v9n2.1114

Silva-Cañaveral, S. (2016). La investigación-creación en el contexto de la formación doctoral en diseño y creación en Colombia. Revista de Investigación, Desarrollo e Innovación, 7 (1), 49-61. doi: https://doi.org/10.19053/20278306.v7.n1.2016.5601

Stoner, J. A. F., Freeman, R. E., \& Gilbert, D. R. (1996). Administración. México: Prentice Hall.

Vásquez-Bustos, Y. A. (2013). La gestión del talento humano, estrategia clave para la organización (Trabajo de especialización). Bogotá, Colombia: Universidad Militar Nueva Granada. 
Vergara-Arrieta, J. J. (2015). Los sistemas de medición del desempeño estratégico frente a la competitividad y sustentabilidad. Saber, Ciencia y Libertad, 10 (2), 17-26. Recuperado de: https://dialnet. unirioja.es/servlet/articulo?codigo $=5295019$
Vergara-Arrieta, J., \& Carbal-Herrera, A. (2014). Diseño de un sistema de gestión en responsabilidad social empresarial para pequeños hoteles en la ciudad de Cartagena. Saber, Ciencia y Libertad, 9 (2), 91-108. doi: http://dx.doi.org/10.22525/sabcliber.2014v9n2.91108 